\title{
Signaling Character in Electoral Competition
}

\author{
By Navin Kartik and R. Preston McAfee*
}

\begin{abstract}
We study a one-dimensional Hotelling-Downs model of electoral competition with the following innovation: a fraction of candidates have "character" and are exogenously committed to a campaign platform; this is unobservable to voters. Character is desirable, and a voter's utility is a convex combination of standard policy preferences and her assessment of a candidate's character. This structure induces a signaling game between strategic candidates and voters, since a policy platform affects voters' utilities not only directly, but also indirectly through inferences about a candidate's character. The model generates a number of predictions, starting with a failure of the median voter theorem. (JEL D72, D82)
\end{abstract}

Americans believe Mr. Bush himself honestly believed Saddam was a threat ... [voters] can tell he is not doing it all by polls and focus groups. ... You can agree or disagree with him, but it is hard to doubt his guts, his seriousness, and his commitment. ... This is why in presidential elections character trumps everything.

-Wall Street Journal editorial, April 22, 2004

Throughout the 2000 US presidential race, spokespersons for the George W. Bush campaign frequently alleged that opponent Al Gore would "say anything to get elected." The implicit suggestion is that George Bush believed in his position and would state it even if it hurt his chances of being elected. The interesting proposition the Bush campaign took is that voters should vote against politicians who state only popular positions, because such politicians lack character.

The idea that politicians might lack character is not new. Criticism of career politicians and insiders has been a frequent refrain in political

\footnotetext{
* Kartik: Department of Economics, University of California, San Diego, 9500 Gilman Drive, La Jolla, CA 92093-0508 (e-mail: nkartik@ucsd.edu); McAfee: Division of the Humanities and Social Sciences, California Institute of Technology, Pasadena, CA 91125 (e-mail: preston@mcafee.cc). We thank Nageeb Ali, Ethan Bueno de Mesquita, Steve Callander, Arnaud Costinot, Vince Crawford, Dino Gerardi, Nir Jaimovich, Dilip Mookherjee, Joel Sobel, and various audiences for comments and discussions; three anonymous referees for helpful suggestions; and Jeremy Bulow for encouraging us to develop a model of primaries.
}

campaigns. George Washington said, "Few men have virtue to withstand the highest bidder." Calvin Coolidge said, "Character is the only secure foundation of the state." Barry Goldwater used character ineffectively against Lyndon Johnson in 1964. In a series of television advertisements, a spokesman said, "You must not give power to a man unless, above everything else, he has character. Character is the most important qualification the president of the United States can have." It is a historical irony that Goldwater's spokesman for presidential character was Richard Nixon.

The simple purpose of this paper is to investigate the effects of character-taken to be an exogenous characteristic of people-on policy selection and voting. The idea is that a politician with character prescribes to voters the position that the politician thinks is best. Politicians without character tell voters whatever is most likely to get the politician elected. Voters have preferences over both positions and character. Voters try to infer character from the positions taken by candidates. Only the candidates without character are strategic, because the candidates with character state their exogenous best position.

One immediate consequence of character is that the median voter theorem cannot hold. ${ }^{1}$ To see why, suppose that all politicians without character choose the preferred position of the

\footnotetext{
${ }^{1}$ Throughout, we use the term "median voter theorem" in its strong sense of policy convergence, referring to when all candidates without character choose the median voter's ideal policy as their platform.
} 
median voter. Then, even the slightest deviation from that position will inform the voters that the candidate has character, and hence insure the election of the candidate, who would be a politician with character almost at the median. This is by itself an important effect of character, because while the median voter theorem is robust in many theoretical models (see, for example, Jeffrey S. Banks and John Duggan 2005), it does not appear to be an empirically salient characteristic of many elections (e.g., Stephen Ansolabehere, James M. Snyder, Jr., and Charles Stewart III 2001).

The theory we develop produces a unique equilibrium, in which candidates without character randomize. The distribution of their positions is related to the distribution of the positions of candidates with character, with a distortion toward the median. Thus, even though the median voter theorem fails in its strong form, policies are biased toward the center. Most elections result in a tie; only extreme candidates lose, and extremists are candidates with character. Tied elections arise because candidates without character randomize, randomization entails indifference, and in the political context, indifference entails ties.

Why do voters care about character? We appeal to a standard answer given in the literature: voters use it to partially assess the set of actions a candidate may take if elected. Politicians cannot commit to a full set of contingent actions, and so voters are necessarily unsure what an elected official will actually do once in office. At most, the candidate can commit to a handful of stated positions. As voters typically care about subtle and sometimes unobservable behavior by an elected official, voters will also care about attributes of the candidate beyond the candidate's stated policy dimension. Canonical attributes of this kind are known as valence and are familiar characteristics of political theory (Donald E. Stokes 1963). ${ }^{2}$ The crucial feature of valence characteristics is that they are monotonically valued by all voters-more of the trait is preferred to less-independent of ideological

\footnotetext{
${ }^{2}$ Not all valence attributes are about character, however. Although Stokes's (1963) original discussion of valence comports well with our notion here, more recent literature sometimes uses the term valence to describe attributes such as "handshaking ability," charisma, and so forth. These traits are not what we have in mind.
}

position. It is this interpretation we take when introducing our basic model. The novel aspect of our approach is that the position chosen by the candidate is interpreted by the voters as a signal about character.

In the latter part of the paper, we discuss how our analysis can be extended to cover a richer set of voter preferences. In particular, this permits voters with differing ideal policy positions to value a candidate's character differently. Moreover, a voter's preference for character can vary with the stated position of the candidate. This allows us to endogenize the preference for character, stepping beyond mere valence interpretations. Suppose that an elected official takes actions of two kinds: observable or "in plain view," and unobservable or "out of sight." Candidates can credibly commit to what observable actions they will take because voters can punish them should they not keep their promises; on the other hand, unobservable actions cannot be committed to, since voters cannot enforce such promises. A politician who has character says what he will actually do (on both dimensions), whereas a politician without character may do something very different on the unobservable dimension from what he promised-and necessarily lives up to- on the observable dimension. In this sense, it is natural to interpret the character trait here as that of integrity. ${ }^{3}$ This setting generates an endogenous taste for character among voters, which will depend both on stated position of the candidate and a voter's own ideal position. Under some assumptions, our main insight extends to such an environment.

Recent literature, such as Martin J. Osborne and Al Slivinski (1996) and Timothy Besley and Stephen Coate (1997), considers candidates who cannot commit to positions at all. Lack of commitment should tend to make candidate character more important, strengthening the implications of our analysis. Some commitment is plausible, however, based on repeated game arguments of Alberto Alesina (1988) and Alesina and Stephen E. Spear (1988); empirical

\footnotetext{
${ }^{3}$ The Merriam-Webster Dictionary definition of "integrity" is "firm adherence to a code of especially moral or artistic values." Discussion of integrity often arises in politics and, in fact, the word was the most-researched word on Merriam-Webster online by Americans in 2005 (CNN, http://www.cnn.com/2005/US/12/10/top.word.ap.ap/).
} 
support for commitment appears in Keith T. Poole and Howard Rosenthal (1997). One implication of our analysis is that candidates who change their position toward the median may be perceived as lacking character, thus encouraging commitment to positions. Thus, in a dynamic or repeated election context, the possibility of character itself may enhance commitment.

We formulate our model in the simplest possible structure: the standard one-dimensional model of electoral competition following Anthony Downs (1957), adapted from the spatial model of Harold Hotelling (1929). Character itself is taken to be an exogenous characteristic of candidates. We think it is reasonable to consider that character is formed long before individuals choose to enter politics, and that character in the general population is in fact exogenous. Character may, however, play a role in the selection of candidates who run for office (e.g., B. Douglas Bernheim and Kartik 2004), and a weakness of our analysis is that the process generating candidates is not modeled. In defense, we analyze the subgame of platform selection given an arbitrary candidate selection mechanism, and analysis of the subgame is necessary to investigate a more general model.

We find that the posterior probability that a candidate has character is higher the further the candidate is from the ideal position of the median voter. This is the feature of the equilibrium that leads to voter indifference. Voters think extremists usually mean what they say, while middle-of-the-road candidates are more likely simply to have said what voters want to hear. The beliefs are constructed to create indifference among candidates without character. A useful aspect of this construction is that it leads to a closed form for the density of positions of candidates without character, up to a single parameter that must be implicitly constructed. This construction would aid an attempt at empirical analysis, although the problems of quantifying the position space and beliefs about character necessary for empirical testing are daunting, indeed.

As the proportion of candidates with character diminishes to zero, the equilibrium platform distribution of those without character collapses on the median voter's ideal point. The support of these positions, however, does not collapse. Interestingly, if almost all candidates have char- acter, both the distribution and support of positions of candidates without character also collapses to the median. Thus, while there is a sense in which the median voter theorem holds at either extreme of the model, the mere presence of character has an echo on policy platforms even when it is very unlikely.

An important feature of the equilibrium of our model is that it is an ex post equilibrium, which means that even after the candidates see each other's position, they do not regret their choice. Thus, it does not matter if the game is played sequentially or simultaneously, as the predicted behavior is invariant to timing. We find this a particularly appealing aspect of our mixed-strategy equilibrium.

The theory presented here provides a theoretical but intuitive grounding for a mechanism that is often believed to operate in real elections. As the opening quote from the Wall Street Journal suggests, the perception of not pandering to the public can be valuable to a politician. This provides a novel explanation of why candidates may select nonmedian platforms and, moreover, why voters may vote for such candidates, rather than simply selecting a centrist candidate. Applications of this principle abound. For example, it is widely agreed that in the 2004 US presidential election, George W. Bush won despite choosing a platform that was well to the right of the center. His victory is often attributed to a belief among the public of his "conviction" in his policy position, in contrast to the perception of John Kerry. This is consistent with our theory. Moreover, the model delivers this as rational behavior and, in particular, rational inferences by the public. However, our theory also makes the following point: the noncentrist candidate may not truly possess the desirable character trait; he could, in fact, be a strategic politician mimicking the behavior of those with character.

As another example, consider the case of the Flemish Liberal Party (VLD) in Belgium. ${ }^{4}$ In 1994, the VLD committed to propose as its platform the policy preferred by the majority of the Belgian public, which it elicited through a public poll. It lost the election by a large margin to a less centrist party. In 1999, on the other

\footnotetext{
${ }^{4}$ We owe this example to Juan D. Carrillo and Micae Castanheira (2002).
} 
hand, the VLD did not pursue this approach, and instead proposed a less centrist platform without the same kind of public poll. It won the election, beating more centrist parties. Why? The explanation here is that in simply pandering to the median voter in 1994, the VLD leaders signaled a lack of character. In contrast, in 1999 , despite not being as desirable purely on the platform dimension, the new leaders of the VLD signaled that they would choose policies that they believed were best, and this trait was valued by voters. Needless to say, this should be viewed as just one component in explaining the reversal in the VLD's fortunes.

As a final example, we recall a well-publicized statement made by senator John McCain during a 1999 Republican primary debate in Iowa: "I'm here to tell you the things that you don't want to hear." McCain went on to denounce ethanol subsidies, which are widely popular in Iowa. From the perspective of appealing to voters on policy alone, this is perhaps puzzling; in fact, all the other Republican candidates either supported or expressed neutrality on this issue. When a campaign is interpreted as also signaling character, however, McCain's approach is straightforward to interpret. The quoted statement prefacing his position suggests that his goal was to convince voters that he would not merely choose platforms that appeal to them, but instead would choose platforms that he truly believed in-and that such a trait should be appreciated by voters.

There are other papers in the literature that derive policy divergence results, starting with Randall Calvert (1985) and Donald Wittman (1977). The closest in spirit to ours is the independent and earlier work of Steven Callander (2005) and Callander and Simon Wilkie (2005). Callander (2005) presumes that voters value a trait about politicians that is unobservable but may be signaled in their campaign platform, with the specific trait being effort devoted to policy implementation. The campaign platform is a commitment, whereas effort cannot be committed to. Unlike our model with character, all candidates in his model are fully strategic; they differ only in whether they are policy-motivated and exert high effort if elected, or office-motivated and exert low effort if elected. Callander and Wilkie (2005) study a model where there is no commitment to policy (and no issue of effort exerted in office), so that every candidate will implement his ideal position if elected. Candidates, however, can be one of two types: either they suffer a zero disutility from lying about their preferred position, or they suffer some finite cost of lying (as in Banks 1990), which increases with the magnitude of their lie.

While there are obvious similarities in motivation and the results derived here with both Callander (2005) and Callander and Wilkie (2005), ${ }^{5}$ there are important distinctions. The most significant difference is that because candidates with character in our model are not strategic about their platform choice (or, alternatively, suffer an infinite disutility from lying), our main result is a unique equilibrium characterization. In contrast, belief-based refinements of signaling equilibria play a role in both Callander (2005) and Callander and Wilkie (2005). Moreover, as noted above, Callander and Wilkie (2005) assume a lack of policy commitment. Our focus here is on a setting with commitment, although we show how some of central insights can be extended to the case without commitment. In Callander (2005), candidates have preference over final policy outcomes and not directly over campaign platforms, whereas our candidates with character can be interpreted as having direct preferences over platforms. Thus, we believe our notion corresponds more closely to the familiar sense of character. We elaborate on this point after introducing the model.

The impact of valence on policy platforms was formally studied by Ansolabehere and Snyder (2000), Enriqueta Aragones and Thomas R. Palfrey (2002), and Timothy Groseclose (2001). In a related vein, John Londregan and Thomas Romer (1993) study an incumbency advantage model where an incumbent has a valence advantage of "ability" over challengers. These papers take a candidate's valence attribute as observable; hence there is no signaling element. Policy divergence stems from asymmetry of valence across candidates. Our divergence

\footnotetext{
${ }^{5}$ In particular, both Callander (2005) and Callander and Wilkie (2005) obtain some results with the following properties: (a) the median voter may not vote for the closest candidate; (b) the less desirable type of politicians play a mixed strategy allocating greater weight on more central positions; (c) the median voter is indifferent over (most) platforms; and (d) more extreme candidates are perceived with increasing probability to be the more preferred type.
} 
result arises even when candidates are completely ex ante symmetric.

The rest of the paper is structured as follows. We present the basic model in Section I. In Section II, we derive the unique equilibrium and develop a number of its implications. Section III studies the impact of character in a two-stage electoral process: party primaries followed by a general election. We briefly discuss some extensions in Section IV, although the details of these extensions are presented separately in an online Appendix (available at http://www.e-aer. org/data/june07/20060106_app.pdf). Section V concludes and provides some directions for future research. Formal proofs are collected in the Appendix.

\section{The Model}

The basic element of the model is a standard Hotelling-Downs one-dimensional policy location game. The set of policies is denoted $X=[0$, 1]. There is a continuum of voters (synonymous with citizens), each with single-peaked policy preferences on $X$. A voter is identified by her ideal point, ${ }^{6} v \in X$, and we assume that her policy preferences can be represented by a utility function $u(x, v)$ that is twice continuously differentiable, and satisfies $u_{1}(v, v)=0$ and $u_{12}(x, v)>0$ for all $x .^{7}$ The median ideal point among voters is $m \in(0,1)$. There are two candidates (synonymous with politicians), $A$ and $B$, each of whom must commit to a platform, $x^{i} \in$ $X$. After observing both candidates' platforms, each voter votes sincerely to maximize her expected utility. ${ }^{8}$ The candidate with greater vote

\footnotetext{
${ }^{6}$ Throughout, we use female pronouns for voters and male pronouns for candidates.

${ }^{7}$ This represents single-peaked preferences because for any $x \neq v$,

$$
\begin{aligned}
u(v, v)-u(x, v) & =\int_{x}^{v}\left[u_{1}(z, v)-u_{1}(z, z)\right] d z \\
& =\int_{x}^{v} \int_{z}^{v} u_{12}(z, y) d y d z>0 .
\end{aligned}
$$

${ }^{8}$ Sincere voting is fully rational in this setting. It serves only to eliminate trivial outcomes such as all voters voting for one candidate, and no voter deviating because each individual is powerless to change the outcome.
}

share wins the election; ties are resolved by a fair coin toss.

We now depart from the standard model by introducing character as follows. Character is a binary variable: candidate $i \in\{A, B\}$ either possesses it $\left(c^{i}=1\right)$ or does not $\left(c^{i}=0\right)$. This is private information and drawn independently from a Bernoulli distribution with $\operatorname{Pr}\left(c^{i}=1\right)=$ $b>0$. If a candidate $i$ has character, his platform choice, $x^{i}$, is constrained to be the draw of a random variable that has a differentiable cumulative distribution function $F$ with density $f(x)>0$ for all $x \in X .{ }^{9}$ Hence, a candidate with character has no strategic choice to make, and we refer to such types as nonstrategic types. On the other hand, candidates without character care only about holding office, and hence strategically choose their platform to maximize their probability of being elected.

Voters care about character in addition to policy: a voter $v$ 's expected utility from a candidate $i$ with platform $x^{i}$ is denoted $U\left(x^{i}, v\right)$, where

$$
U\left(x^{i}, v\right) \equiv \lambda \operatorname{Pr}\left(c^{i}=1 \mid x^{i}\right)+u\left(x^{i}, v\right) .
$$

Thus, $\lambda>0$ is the relative weight voters attach to a politician's character. Note that the inference about a politician's character depends upon his chosen platform. The standard HotellingDowns model is a special case of our model when either $b=0$ or $\lambda=0$.

Some remarks are in order about our modeling choices, and further literature connections. First, candidates without character in our model are purely office-motivated and fully strategic. Formally, candidates with character are nonstrategic and can be thought of as "crazy" types, following David M. Kreps et al. (1982). This interpretation is strained, however, and we prefer to think of them as fully rational, despite our choice of terminology. The idea is that candidates with character suffer (infinite) disutility from proposing a platform they do not "believe in." This notion has similarities with what John E. Roemer (1999) calls militant motivation. As discussed in the introduction, our assumption that citizens care about a pol-

\footnotetext{
${ }^{9}$ The assumption that $F$ has full support is not crucial Our analysis extends to arbitrary distributions; failure of the median voter theorem (Corollary 1 ) requires only that the support of $F$ includes an interval containing the median, $m$.
} 
itician's character is meant to capture the idea that citizens not only have policy preferences over the campaign issues, but also care about some unobservable characteristic of politicians which is correlated with their willingness to pander in order to gain office. Indeed, our model can be thought of as a reduced form for the following: each candidate $i$ has a policy, $p^{i}$, drawn from the $\operatorname{cdf} F$, that he thinks is "right." He then learns whether he has character, in which case he necessarily chooses platform $x^{i}=p^{i}$, perhaps due to a preference for not pandering. If he does not have character, he strategically chooses platform $x^{i}$ to maximize his probability of being elected. Now, suppose character types will resist special interest groups if elected, whereas noncharacter or flexible types will fall prey to them. Voters get a disutility of $\lambda$ from an elected politician who deals with special interest groups. In this environment, a voter has an instrumental reason to prefer politicians who are nonstrategically choosing their platform, all else equal.

The candidates with character in our model are not policy-motivated in the usual sense the term is used (Calvert 1985; Wittman 1977). The utility for policy-motivated candidates depends on the final policy outcome and not directly on their individual policy platform, whereas candidates with character in our model care directly about the platform they propose, regardless of the final policy outcome. In a sense, character candidates are procedurally motivated. To take another perspective, policy-motivated candidates have unlimited ability to compromise in platform, and will do so if it results in a more desirable final policy outcome; character candidates are limited in their ability to compromise on their platform. Morris P. Fiorina (1999, 9, fn. 10) makes this distinction.

We have assumed that the prior probability of having character and the distribution of platforms conditional on character is the same across both candidates. Thus, there is no ex ante asymmetry between the candidates. This contrasts with the literature on observable valence asymmetry (e.g., Aragones and Palfrey 2002). In Section IV, we discuss ex ante differences between the candidates.

Since candidates with character are nonstrategic, we refer to a candidate's strategy as his behavioral rule conditional on not having character, i.e., conditional on being strategic. A strategy for candidate $i \in\{A, B\}$ is represented by a cumulative distribution function (cdf), $G^{i}$. If $G^{i}$ has a density, we denote it $g^{i}$. As this is a signaling game, voter beliefs about a candidate's character are critical. Let $\varphi^{i}(x)$ be the posterior probability that $i$ has character given his platform choice of $x$.

It is convenient to define $\mu(x) \equiv u(x, m)$, so that $\mu(x)$ is the median voter's policy utility from platform $x$. Given the posterior belief of character and the platform of candidate $i$, the median voter's expected utility should this candidate be elected is

$$
\alpha^{i}\left(x \mid \varphi^{i}\right)=\lambda \varphi^{i}(x)+\mu(x) .
$$

Where there is no risk of confusion, we typically suppress the dependence of $\alpha^{i}$ on $\varphi^{i}$ to reduce notation. Sincere voting implies that candidate $i$ wins and candidate $j \neq i$ loses if $\alpha^{i}\left(x^{i}\right)>\alpha^{j}\left(x^{j}\right)$. When the two expressions are equal, the election is tied, and each candidate gets elected with probability $1 / 2$. Given the beliefs $\varphi^{A}$ and $\varphi^{B}$, voter behavior is completely pinned down (except perhaps for a measure 0 set of voters), hence we are not explicit about it in what follows.

Our solution concept is that of (weak) perfect Bayesian equilibrium (Drew Fudenberg and Jean Tirole 1991). This requires that the platform distributions, $G^{A}$ and $G^{B}$, respectively, maximize the probability of being elected for each strategic candidate given voter beliefs $\varphi^{A}$ and $\varphi^{B}$, and that $\varphi^{A}$ and $\varphi^{B}$ be consistent with Bayes's Rule. ${ }^{10}$ For technical convenience, we restrict attention to equilibria where the distributions $G^{A}$ and $G^{B}$ can be written as the sum of absolutely continuous and discrete distributions. ${ }^{11}$

\section{Signaling Character}

\section{A. The Unique Equilibrium}

Due to the symmetry in the model, a candidate must win with positive probability if strategic. To see this, observe that a candidate can

\footnotetext{
${ }^{10}$ More precisely, each $\varphi^{i}$ must be a regular conditional distribution derived from the distributional strategy (Paul R Milgrom and Robert J. Weber 1985) induced by $F$ and $G^{i}$.

${ }^{11}$ By the Lebesgue decomposition theorem for the Real line, this is only a restriction in precluding a strategy from having a singular component without mass points.
} 
always play the same strategy as his opponent. If he does so, he loses with probability one only if voters believe that, for all platforms in the support of this strategy, he is less likely to have character than his opponent. Given that both candidates have the same prior likelihood of character, $b>0$, and the same distribution over platforms with character, $F$, this cannot be the case.

The only way that strategic candidates win with positive probability, and yet maximize their probability of winning, is if every platform that they choose offers the same value to the median voter (and all platforms not chosen offer no greater value). Call this value $\alpha^{*}$. Since there are no mass points in the platforms of the candidates with character, strategic candidates will not use mass points either. To see this, note that the Bayes's update on the probability a candidate has character is zero at any mass point in the strategy of the strategic candidates. Thus, at any mass point in the strategy, there is a choice for the strategic candidate with a nearby policy platform, but a positive likelihood of having character. Consequently, the distribution of positions chosen by the candidates will be continuous. This means that we can write the Bayes's update as

$$
\varphi^{i}(x)=\frac{b f(x)}{b f(x)+(1-b) g^{i}(x)} .
$$

Since within the support of their strategies, both strategic candidates offer value $\alpha^{*}$, we conclude that

(1) $\alpha^{*}=\mu(x)+\lambda \frac{b f(x)}{b f(x)+(1-b) g^{i}(x)}$.

The equation above states that for any position that may be taken by a strategic candidate, the utility offered to the median voter is a constant. Recall that this utility is the sum of the position's direct value to the median voter and the Bayes's updated beliefs about the candidate's likely character. Equation (1) can be solved for the density of platform choices, which turns out to be

$$
\begin{aligned}
g^{i}(x) & =g^{*}(x) \\
& =\max \left\{0, \frac{b f(x)}{1-b}\left[\frac{\lambda}{\alpha^{*}-\mu(x)}-1\right]\right\} .
\end{aligned}
$$

This is a symmetric expression, so the platform distribution is the same for both strategic candidates. Moreover, the value of $\alpha^{*}$ is determined by the requirement that $g^{*}$ be a density, and hence integrate to one. It is readily shown that $\mu(m)<\alpha^{*}<\mu(m)+\lambda$, since a candidate can always offer a value of at least $\mu(m)$ by choosing platform $m$, and can offer a value of no greater than $\mu(m)+\lambda$.

An important aspect of this construction is that the median voter is indifferent over every position taken by strategic candidates. Thus, even knowing the opponent's platform, a strategic candidate has no incentive to revise his position. This property means that the equilibrium is an ex post equilibrium, and in this setting, it has two important implications. First, the outcome is not sensitive to the timing of the game: the same behavior remains an equilibrium whether one candidate announces first, or second, or both announce platforms simultaneously. Second, all elections between strategic candidates result in a tie, even though candidates have distinct positions.

It turns out that, with substantially more work, it can be shown that this equilibrium is unique. This conclusion is summarized in Theorem 1, the formal proof of which is in the Appendix.

THEOREM 1: There is a unique equilibrium. It is an ex post equilibrium where both candidates use the same strategy, $G^{*}$, with density

$$
g^{*}(x)=\max \left\{0, \frac{b f(x)}{1-b}\left[\frac{\lambda}{\alpha^{*}-\mu(x)}-1\right]\right\},
$$

where $\alpha^{*} \in(\mu(m), \mu(m)+\lambda)$ is the unique constant such that $\int_{x} g^{*}(x) d x=1$.

Therefore, both candidates mix in equilibrium, using a continuous density. Our interpretation of mixed strategies follows the Bayesian view of opponents' conjectures, originating in John C. Harsanyi (1973). That is, a candidate's mixed strategy need not represent him literally randomizing over platforms; instead, it represents the uncertainty that the other candidate and the electorate have about his pure strategy choice. Each candidate could be playing a pure strategy which depends upon an auxiliary variable that is his private information. 
One implication of Theorem 1 that is worth emphasizing is that the median voter theorem (MVT) does not hold in our model. Note that in the current setting, the appropriate version of the MVT is that, ex ante, each candidate chooses platform $m$ with probability $1-b>0$, viz., with the probability of being strategic.

COROLLARY 1: The MVT fails. In the unique equilibrium, the ex-ante probability that either candidate chooses platform $m$ is 0 .

\section{B. Properties}

We now derive various implications of Theorem 1 . The first is a simple observation.

FACT 1: If a candidate is strategic, he wins with probability at least as large as if he had character.

This is an immediate consequence of the fact that a strategic candidate provides the median voter with utility $\alpha^{*}$, whereas a candidate with character provides the same utility if his platform falls within the support of the equilibrium strategy, $G^{*}$, and strictly less utility if his platform falls outside the support.

Since $\alpha^{*}$ is the expected utility of the median voter, comparative statics on $\alpha^{*}$ immediately become comparative statics on the utility of the median voter. The comparative statics on $\alpha^{*}$ are readily computed from the equation

$$
\begin{aligned}
& \int_{x} \max \left\{0, \frac{b f(x)}{1-b}\right. \\
& \left.\quad \times\left[\frac{\lambda}{\alpha^{*}(b, f, \mu, \lambda)-\mu(x)}-1\right]\right\} d x=1,
\end{aligned}
$$

which implicitly defines $\alpha^{*}$ as a function of $b, f$, $\mu$, and $\lambda$. We use this and the equilibrium construction from the previous section to discuss various implications of our theory. Denote by $\varphi^{*}(x)$ the posterior held by voters upon seeing platform $x$ in equilibrium.

FACT 2: The posterior belief on character is single-troughed around the median. That is, $\varphi^{*}(x)$ is strictly decreasing for $x<m$, and strictly increasing for $x>m$, whereas $g^{*}(x) /$ $f(x)$ is strictly increasing for $x<m$, and strictly decreasing for $x>m$.

This property of $g^{*}(x) / f(x)$ is immediate from inspection of (2), because $\mu$ is single-peaked around $m$. That the reverse is true for $\varphi^{*}$ then follows because we can write the Bayes's update as

$$
\varphi^{*}(x)=\frac{b}{b+(1-b) \frac{g^{*}(x)}{f(x)}} .
$$

Thus, strategic candidates skew their positions toward the median voter's preferred position. Moreover, voters believe that candidates near the median voter's preferred policy are less likely to have character, and the farther away the chosen position is, the more likely the candidate is to have character. This leaves the median voter indifferent, provided the positions are in the support of $g^{*}$. Outside the support of $g^{*}$, the voters are certain the candidate has character but the candidate loses nonetheless, because the advantage of assured character is unable to overcome the disadvantage of an extreme position. Thus, the model accommodates extreme positions losing with certainty and distinct moderate positions resulting in ties.

FACT 3: The median voter's utility from a strategic candidate, $\alpha^{*}$, is increasing in the value of character, $\lambda$, and the probability of character, $b$.

This directly follows from inspection of (3). That ex ante welfare, $\alpha^{*}$, increases in $b$ is relatively trivial, since voters value character, and $b$ is the ex ante probability of a candidate having character. The rationale behind $\alpha^{*}$ increasing in the weight placed on character, $\lambda$, is more subtle. As $\lambda$ rises, $g^{*}(x) / f(x)$ flattens out, and strategic candidates look more like candidates with character. This casts the effect of $\lambda$ on utility in an interesting light. The flattening of $g^{*}(x) / f(x)$ means that the value of the platform offered by strategic candidates falls as $\lambda$ rises. Moreover, the likelihood that a candidate has character hasn't changed. Thus, the value offered by strategic candidates falls and their prevalence remains unchanged. The behavior and prevalence of candidates with character hasn't changed. Thus, the overall value of the system seems to 
have fallen. The apparent paradox is resolved by noting that the total value of the candidates with character has risen because character is valued more highly.

FACT 4: The support of a strategic candidate's choices is increasing in the value of character, $\lambda$.

By (2), the support of $g^{*}$ is given by the solutions to $\lambda+\mu(x) \geq \alpha^{*}$. Thus, whether the support increases in $\lambda$ reduces to the question of whether $\left(\partial \alpha^{*} / \partial \lambda\right)<1$. This is indeed the case, since by differentiating (3), we see that

$$
\begin{aligned}
& \int_{\left\{x \mid \lambda+\mu(x) \geq \alpha^{*}\right\}} \frac{b f(x)}{1-b} \frac{1}{\left[\alpha^{*}-\mu(x)\right]^{2}} \\
& \times\left[\alpha^{*}-\mu(x)-\lambda \frac{\partial \alpha^{*}}{\partial \lambda}\right] d x=0 .
\end{aligned}
$$

Since $\alpha^{*} \leq \lambda+\mu(x)$ for all $x$ in the support of $g^{*}$, the equality above cannot hold unless $\left(\partial \alpha^{*} / \partial \lambda\right)<1$. Thus, the support of $g^{*}$ is indeed increasing in $\lambda$. This means that as character becomes more important, the likelihood of extremists, with and without character, being elected rises. The intuition is that when policy becomes less important relative to character, candidates with extreme platforms can still win. If character is sufficiently important, so that is $\lambda$ is sufficiently large, $g^{*}$ will have full support. In this case, any election is tied.

FACT 5: As utility from character becomes infinitely more important than direct policy utility, strategic candidates perfectly mimic the distribution of candidates with character. That is, as $\lambda \rightarrow \infty, g^{*}(x) \rightarrow f(x)$ for all $x$.

We defer the argument to the Appendix. Intuitively, as the weight on character diverges, the gain from being perceived as having character increases for each candidate. Thus, in the limit, strategic candidates fully mimic the distribution of those with character.

Another comparative static considers a change in the platform of candidates with character. Suppose that $f$ is replaced with $h$, and that $h(x)-f(x)$ is increasing for $x<m$, and decreasing for $x>m$. That is, candidates with character are more likely to come from the center under $h$ than under $f$. In this case, we say that $h$ is a more central density than $f$.

FACT 6: The median voter's utility from a strategic candidate, $\alpha^{*}$, is higher when the distribution of platforms from a candidate with character comes from a more central density.

The proof is in the Appendix. The idea is simple: strategic candidates mimic the distribution of those with character, but skew their play toward the median voter's preferred policy. If the distribution of platforms of candidates of character is more concentrated toward the center, then both strategic and nonstrategic candidates' play becomes more desirable to the median voter in policy terms. Thus, $\alpha^{*}$ goes up.

For our last comparative statics, we consider the limits as $b$ converges to 0 or 1 .

FACT 7: As $b \rightarrow 1, \operatorname{Supp}\left(g^{*}\right) \rightarrow\{m\}$. As $b \rightarrow$ $0, \operatorname{Supp}\left(g^{*}\right) \rightarrow\{x \mid \mu(x)+\lambda \geq \mu(m)\}$, but $G^{*}$ converges to an atom on $m$.

The proof is in the Appendix. If most candidates have character, so that $b$ is close to one, there is little advantage to a strategic candidate of signaling character by position, since the prior is so strong. Consequently, the support of the distribution of the strategic candidate's strategy collapses to $m$. This case replicates the standard model and the MVT emerges at this limit. In contrast, if there are few candidates with character, and $b$ is close to zero, the distribution collapses on $m$ but the support of the distribution converges to the set $\{x \mid \mu(x)+\lambda \geq$ $\mu(m)\}$. This is as large as the support can get for a given $\lambda$. Thus, while almost all strategic candidates locate very near the middle, the possibility of strategic candidates a long way from the middle remains in the limit as candidates without character vanish. The possibility of candidates with character then has an echo in the model, even when the probability of such candidates goes to zero. Note that in such a case, ex post, an elected candidate may have a position far from the middle.

\section{Two-Stage Elections}

In many electoral systems, such as the US presidential process, a candidate must win a party nomination before competing in a general 
election. To a first approximation, a party nomination is won by garnering the majority of votes among the subset of all voters who are affiliated with the party. ${ }^{12}$ This implies that the set of voters a candidate must appeal to is different in the within-party primary vote (first stage) from the general election vote (second stage). For instance, in the United States, the median voter in the Republican (respectively, Democratic) party's primary is to the right (left) of the median voter in the general election. To date, there has been a relative paucity of theoretical work on electoral outcomes in such settings. ${ }^{13}$ In this section, we study a simplified version of our model extended to a two-stage electoral process. Rather than presenting an exhaustive analysis, our goal is to suggest only how our central theme can be extended to such settings, with interesting implications.

For tractability, we simplify the policy space to the discrete set $X=\{-1,0,1\}$. There are two political parties, denoted $L$ and $R$. Within each party, there are two candidates for office. The winner of the election is decided through a two-stage process. In the first stage, the primary election, each candidate from each party starts by simultaneously committing to a policy position that is either 0 or his own party's extreme policy: 1 for party $R$ and -1 for party $L$. Then, each party determines its nominee through a vote between its two candidates by the citizens affiliated with the party. In the second stage, the general election, all citizens get to vote for one of the two nominees (one nominee from each party); the winner of this general election is elected to office and implements the policy to which he committed in the first stage. That is, candidates are committed to the same platform in the general election as they chose in their party primary.

As usual, denote a voter's policy utility by $u(x, v)$, where $x$ is the policy and $v$ is her ideal point. For convenience, assume there is a distinct, single (and thus median) voter in each of the three elections: the two party primaries and

\footnotetext{
${ }^{12}$ Strictly speaking, this is true only for so-called "closed" primaries.

${ }^{13}$ A recent exception is Adam Meirowitz (2005), although his focus is quite different from ours. Elisabeth R. Gerber and Rebecca B. Morton (1998) discuss the impact of "closed" versus "open" primaries, and provide a good reference to some of the earlier formal literature.
}

the general election. ${ }^{14}$ The voter in party $L$ 's primary has ideal point -1 , the voter in party $R$ 's primary has ideal point 1 , and the voter in the general election has ideal point 0 . Furthermore, we restrict attention to a symmetric setup across parties and policies such that $u(-1,0)=$ $u(1,0)<u(0,0), u(0,1)=u(0,-1), u(1$, $-1)=u(-1,1)$, and $u(-1,-1)=u(1,1)$. If a candidate has character, then any voter gets an added utility of $\lambda>0$ if that candidate is elected. Each candidate has probability $b \in(0$, 1) of having character; those with character are committed to an exogenously drawn platform. A candidate with character from the $R$ party draws his platform from a distribution that places probability $f_{0}>0$ on platform 0 and probability $f_{1} \equiv 1-f_{0}>0$ on platform 1 . Symmetrically, a candidate with character from the $L$ party chooses platform 0 with probability $f_{0}$ and platform -1 with probability $f_{-1} \equiv 1-$ $f_{0}$. Candidates without character aim to maximize their probability of being elected to office (i.e., winning the general election).

A key feature of the setting we have described is that, ceteris paribus, an extreme candidate is preferred in the party primaries, whereas a centrist candidate is preferred in the general election. We assume, however, that primary voters are forward-looking insofar as they don't myopically vote for the candidate they prefer in the primary; rather, they take into account the prospects of each candidate in the general election. That is, voters in the primaries vote for the candidate who maximizes their overall expected utility, which depends only on the general election winner's platform and character. In particular, the primary median of each party would prefer a centrist (from either party) over an extremist from the other party, character considerations aside.

We seek symmetric equilibria where strategic candidates from party $R(L)$ play platform 0 with probability $g_{0}$ and platform $1(-1)$ with probability $g_{1} \equiv 1-g_{0}\left(g_{-1} \equiv 1-g_{0}\right)$. By Bayes's rule, the belief about character as function of platform, $\varphi(x)$, is the same for all candidates in a symmetric equilibrium, and is given by $\varphi(x)=\left[b f_{x} / b f_{x}+(1-b) g_{x}\right]$ for $x=-1,0,1$.

\footnotetext{
${ }^{14}$ This can be interpreted as representing median voters among a large set of voters in each election, with one caveat in footnote 16 .
} 
Even within this simple setting, a variety of symmetric equilibria can emerge, sometimes for the same set of parameters. The reason for multiplicity is a kind of "self-fulling prophecy" across primaries and the general election. The candidate-generation process during the primaries influences the success rate during the general election: extreme candidates who emerge from the primary are less likely to do well in the general election, all else equal. The potential for success in the general election, however, influences the voter preferences in the primary: if a candidate with a particular platform is believed to be more likely to win the general election, that candidate is preferred in the primary.

Three kinds of equilibria are of particular interest. The first kind, Naive Policy Preference equilibrium, has the property that in the general election, centrists are preferred over extremists, whereas in the party primaries, extremists are preferred over centrists. ${ }^{15}$ In the second kind, General Election Indifference equilibrium, the general election voter is indifferent between centrists and extremists (and randomizes in voting), whereas in the party primaries, extremists are preferred over centrists. Finally, in the third kind, Centrist Dominant equilibrium, both general election and primary voters prefer centrists over extremists.

The following result shows that under some conditions, Naive Policy Preference and General Election Indifference equilibria exist, whereas Centrist Dominant equilibrium does not. We say that $u$ is convex if $2 u(0,1)<u(1$, $1)+u(-1,1)$, i.e., the party voters prefer a uniform lottery among the two extreme policies over the certain center policy.

THEOREM 2: In the two-stage election model, assume u is convex. Then, Centrist Dominant equilibrium does not exist. Moreover, Naive Policy Preference and General Election Indifference equilibria exist for (possibly distinct) open parameter sets of positive Lebesgue measure.

\footnotetext{
${ }^{15}$ Note that regardless of how strategically candidates behave, there is always positive probability that both candidates in a party's primary have centrist or extreme platforms. Thus, in the general election, there is positive probability on all possible platform pairs by the two nominated candidates.
}

In both the Naive Policy Preference equilibrium and the General Election Indifference equilibrium we construct, strategic candidates randomize between their own party's extreme platform and the center platform. It is important to note that in the General Election Indifference equilibrium, even though the general election voter is indifferent across all candidates, her indifference cannot be broken by randomizing uniformly across centrists and extremists. Instead, since extremists defeat centrists in the party primary, the probability that a centrist beats an extremist in the general election needs to be such that strategic candidates are indeed indifferent between choosing extreme and center platforms at the outset. ${ }^{16}$

Theorem 2 reveals that character can have a powerful effect in two-stage elections, because character permits a General Election Indifference equilibrium to exist for some parameters. In contrast, when $b=0$, such an equilibrium does not exist, because without character, the general election voter is never indifferent between centrist and extreme candidates. ${ }^{17}$ The indifference when $b>0$ arises precisely because even though the general election voter prefers centrist platforms over extreme ones in direct policy utility, the behavior of strategic candidates is biased toward the center, so that extremists are more likely to have character. In this sense, the General Election Indifference equilibrium captures and extends the main insight of our earlier analysis.

\section{Discussion}

We now briefly discuss a few extensions of our theory, with regards to the basic model developed in Section I for one-stage elections. Details and formal results are available in the working paper version of this article, and in the Supplementary

\footnotetext{
${ }^{16}$ With the assumed single voter in the general election, this presents no conceptual issue. If, instead, this were a median voter representing a large number of voters with heterogeneous ideal points, we take the interpretation that the nonuniform tie-breaking results from uncertainty about the median that is resolved between the primary and general election.

${ }^{17}$ When $b=0$, a Centrist Dominant equilibrium always exists. If $b=0$ and $u$ is convex, however, this equilibrium entails each party primary voter playing a weakly dominated strategy, whereas a Naive Policy Preference equilibrium exists in undominated strategies.
} 
Appendix on the AEA Web site (http://www. e-aer.org/data/june07/20060106_app.pdf).

\section{A. Tied Elections}

As already noted, our simple model results in all elections between strategic candidates being tied. Moreover, if the weight on character, $\lambda$, is large enough, then all elections end in ties. This feature can be mitigated by introducing uncertainty about the median voter's location that gets resolved only after the candidates choose platforms. So long as candidates do not possess private information about the median voter's location, it is straightforward to extend our analysis.

\section{B. Ex Ante Asymmetry}

Suppose that the prior likelihood of having character can differ across candidates and, moreover, the distribution of policies conditional on character can also differ. This may be appealing when thinking of candidates as representing different political parties, for example. A generalized version of Theorem 1 applies in such an ex ante asymmetric setting. In particular, there is an ex post equilibrium with candidate-specific strategies analogous to equation (2). It is important to note, however, that ex ante asymmetries can endow one candidate with an advantage resulting in his winning with probability one when strategic. The intuition is related to models with observable valence asymmetries (Aragones and Palfrey 2002; Groseclose 2001).

\section{Richer and Endogenous Preferences for Character}

We have modeled the preference for character as being uniform across all voters and independent of the policy platform, thus represented by a constant $\lambda$. Our analysis can be extended under some conditions, however, to a setting where the preference weight on character depends on both the platform and a voter's personal ideal point. That is, utility for a voter with ideal point $v$ facing a candidate $i$ with policy $x^{i}$ may be given by $U\left(x^{i}, v\right) \equiv \lambda\left(x^{i}, v\right) \operatorname{Pr}\left(c^{i}=\right.$ $\left.1 \mid x^{i}\right)+u\left(x^{i}, v\right)$. One motivation for such a specification is to capture the idea that politicians take actions of two kinds: observable, or "in plain view," and unobservable, or "out of sight." The $u(\cdot, \cdot)$ component of a voter's utility represents the utility over observable actions that have been committed to by the politician during the electoral process. The $\lambda(\cdot, \cdot)$ component represents the voter's utility over the unobservable actions that the politician will take in office. If the politician has character, his position on the unobservable dimension will be the same as what he committed to on the observable action, since those with character say what they will actually do. If he does not have character, however, he might do something very different on the unobservable dimension from what he promised (and necessarily lives up to) on the observable dimension. In this setting, it is natural that the preference for character depends on a candidate's policy position and a voter's ideal point. For example, a voter with ideal point $v=$ 1 may prefer a candidate with platform $x^{i}=0$ not to have character and thus likely do something quite different on the unobservable dimension from what was promised. On the other hand, the same voter may prefer a candidate with $x^{i}=1$ to in fact have character, thus guaranteeing that he will take the same policy position on the unobservable dimension. This preference ordering over character can be reversed for a voter with ideal point $v=0$.

\section{No Commitment}

Finally, suppose that campaign statements are pure cheap talk. If elected, a politician need not necessarily implement his campaign platform, and can instead choose any policy in the policy space. The policy a candidate will actually implement if elected is private information, and drawn from the density $f(x)$. A candidate also has character with probability $b$; this, too, is private information. Candidates with character announce precisely what they will do if elected, whereas candidates without character will say anything to get elected. Voters have preferences only over final policy, $u(x, v)$, and not directly over character at all. Our main result can be extended to such a setting, under some conditions on the primitives. Character becomes endogenously desirable here because a voter with ideal point $v$ would most prefer a candidate who not only proposes platform $v$ but also does so with character. 


\section{Conclusion}

In a president, character is everything. -Peggy Noonan

This paper develops a theory of character in elections. The two key assumptions we make are that some candidates may have character and do not strategically choose policy platforms simply to maximize the probability of getting elected, and voters value character in addition to campaign promises. Character quashes the median voter theorem, as strategic candidates pretend to have the positions of candidates with character. Elections between strategic candidates are tied in the symmetric version of our model. As character becomes more important to voters, the behavior of strategic candidates shifts farther away from the ideal policy of the median voter and closer to the behavior of candidates with character.

We have illustrated a number of extensions of the basic idea. In discussing extensions to richer preferences, we sketched a model of endogenous preference for character, where character entails following the promised policy in all circumstances, even when the choice is unobservable to voters. Voters who care about the unmonitored behavior will naturally care about the character of candidates. Our treatment of this setting is very stylized, however, and it would be useful to model this in a more detailed way. We also believe that the model of twostage elections developed here is worthy of further study in its own right.

An interesting avenue for future research would be to consider the impact of character when a politician makes repeated choices while holding office. If there is a stochastic element over time to the location of the median voter, then the presence of candidates with character may induce rigidity or stability of position, even among candidates without character, for strategic reasons. Thus, even purely office-motivated candidates may appear to be driven by ideology (cf. Joseph E. Harrington 1998). This is intriguing given the empirical evidence on the ideological consistency of elected officials (e.g., Poole and Rosenthal 1997).

A related issue is that we have modeled the proportion of candidates possessing character as an exogenous parameter, but in fact candidates usually run for higher office only after a complex winnowing process that includes serving for lower offices. Does this process tend to favor candidates with character or strategic candidates? In our model, the probability that a candidate with character is elected is no more, and may be strictly less, than the probability that a strategic candidate is elected (see Fact 1). This suggests that the winnowing process may favor strategic candidates. If, however, candidates with character are more likely to be reelected or advance to higher office-perhaps because a candidate's type may be discovered with some probability once in office-then character may actually increase through the political hierarchy. A richer analysis is needed to illuminate the dynamics of candidate selection, but we view our model as a useful starting point.

\section{ApPendix: Proofs}

To prove Theorem 1, some preliminaries are needed. Note that, due to the continuum of policy locations, various statements about optimal strategies will be subject to "almost all" qualifiers; we suppress such caveats unless essential.

LEMMA A.1: In any equilibrium, the strategic type of a candidate wins with positive probability when competing against the strategic type of the other candidate.

\section{PROOF:}

Without loss of generality, it suffices to show that for some set of $Y$ of positive $G^{A}$-measure, $\varphi^{A}(x) \leq \varphi^{B}(x)$ for $G^{A}$-a.e. $x \in Y$. (Because then by concentrating mass on $Y$, strategic $B$ can win with positive probability against strategic $A$.) This is immediate if $G^{A}$ has atoms because $\varphi^{A}(x)=0$ for any $x$ that $G^{A}$ has an atom at, so suppose that $G^{A}$ is atomless, hence absolutely continuous with density $g^{A}$. Let $X^{B}$ be the set of all nonatomic points of $G^{B}$; since $G^{B}$ can have only a countable number of atoms, $X^{B}$ has full $G^{A}$-measure and there is a density of $G^{B}$, denoted $g^{B}$, on $X^{B}$. Then, for a.e. $x \in X^{B}, \varphi^{i}(x)=$ $\left[b f(x) / b f(x)+(1-b) g^{i}(x)\right]$, and there must be a set $Y \subseteq X^{B}$ with the desired properties; otherwise, $1=\int_{X^{B}} g^{A}(x) d x<\int_{X^{B}} g^{B}(x) d x \leq 1$, a contradiction.

We now define an ex post equilibrium, which is an equilibrium such that even if a candidate 
observed his opponent's platform before choosing his own, he would have no incentive to deviate from his prescribed strategy.

DEFINITION 1: An equilibrium with $c d f^{\prime} s G^{A}$ and $G^{B}$ is an ex post equilibrium if the probability that candidate $i \in\{A, B\}$ wins is the same for all realizations of $x^{A}$ and $x^{B}$ in the support of $G^{A}$ and $G^{B}$, respectively.

Given Lemma A.1, it is straightforward that a pair of cdf's $\left(G^{A}, G^{B}\right)$ constitutes an ex post equilibrium strategy profile if and only if for each $i \in\{A, B\}$ :

$$
\begin{array}{r}
\alpha^{i}(y) \leq \alpha^{i}(x) \quad \text { for all } x \in \operatorname{Supp}\left[G^{i}\right] \\
\text { and } y \notin \operatorname{Supp}\left[G^{i}\right] .
\end{array}
$$

An important consequence is that every equilibrium is an ex post equilibrium.

LEMMA A.2: Any equilibrium is an ex post equilibrium.

\section{PROOF:}

The following notation will be used: $\overline{x^{i}} \in \arg$ $\max _{x} \alpha^{i}\left(x \mid \varphi^{i}\right), \overline{\alpha^{i}} \equiv \max _{x} \alpha^{i}\left(x \mid \varphi^{i}\right), \quad \underline{x}^{i} \in \arg$ $\min _{x} \alpha^{i}\left(x \mid \varphi^{i}\right)$, and $\underline{\alpha}^{i} \equiv \min _{x} \alpha^{i}\left(x \mid \varphi^{i}\right)$. First, we prove that condition (A-1) must hold in any equilibrium. It suffices to show that for $i \in\{A$, $B\}, \overline{\alpha^{i}}=\alpha^{i}$, and $\overline{\alpha^{A}}=\overline{\alpha^{B}}$. The latter is straightforward: if not, without loss of generality say $\overline{\alpha^{A}}$ $>\overline{\alpha^{B}}$, then playing $\overline{x^{A}}$ guarantees election for $A$, contradicting equilibrium mixing and Lemma A.1. Similarly, $\underline{\alpha}^{A}=\underline{\alpha^{B}}$. Now, if $\overline{\alpha^{i}}>\underline{\alpha}^{i}$, then $\underline{x}^{i}$ loses at least against $\overline{x^{j}}$, whereas $\overline{x^{i}}$ would win with probability $1 / 2$ against $\overline{x^{j}}$ and moreover against anything that $x^{i}$ either ties or beats, so it is not optimal for $\underline{x}^{i}$ to be in the support of $i$ 's strategy.

Condition (A-2) is also necessary in any equilibrium because if it does not hold, then given condition (A-1), one of the candidates can profitably deviate to winning the election with probability 1 , since he wins only with lower probability in equilibrium by Lemma A.1.
Lemma A.2 implies that candidates do not use mass points in their strategies.

LEMMA A.3: In any equilibrium, both $G^{A}$ and $G^{B}$ are atomless.

\section{PROOF:}

Suppose that $G^{i}$ has an atom on $\hat{x}$. Then $\alpha^{i}(\hat{x})=\mu(\hat{x})$. Since there can be only a countable number of atoms, we can find a small $\varepsilon \neq$ 0 such that

$\alpha^{i}(\hat{x}+\varepsilon)=\lambda \varphi^{i}(\hat{x}+\varepsilon)+\mu(\hat{x}+\varepsilon)>\mu(\hat{x})$.

If $\hat{x}+\varepsilon \in \operatorname{Supp}\left[G^{i}\right]$, condition (A-1) is violated; if $\hat{x}+\varepsilon \notin \operatorname{Supp}\left[G^{i}\right]$, condition (A-2) is violated. Either way, $G^{i}$ cannot be an equilibrium strategy.

Accordingly, any equilibrium strategy, $G^{i}$, has a density, $g^{i}$, and by Bayes's rule,

$$
\varphi^{i}(x)=\frac{b f(x)}{b f(x)+(1-b) g^{i}(x)} .
$$

The next lemma shows that the support of a candidate's strategy must be in an interval containing the median voter's ideal policy.

LEMMA A.4: In any equilibrium, for $i \in\{A$, $B\}, \operatorname{Supp}\left[G^{i}\right]$ is an interval that contains $m$.

\section{PROOF:}

First we argue that the support is an interval. Suppose not for player $i$. Then there exist $x>$ $y>z$ such that $g^{i}(x)>0, g^{i}(y)=0$, and $g^{i}(z)>$ 0 . This implies that $\varphi^{i}(x)<1, \varphi^{i}(y)=1$, and $\varphi^{i}(z)<1$. If $y \geq m$, it follows that $\alpha^{i}(y)>$ $\alpha^{i}(z)$; if $y \leq m$, then $\alpha^{i}(y)>\alpha^{i}(x)$. Either case contradicts condition (A-2) for an ex post equilibrium.

Next, we show that the interval must contain $m$. If it didn't, without loss of generality say it is $[l, h]$ with $h<m$, then $\varphi^{i}(m)=1$, hence for any $x \in[l, h]$,

$$
\alpha^{i}(m)=\lambda+\mu(m)>\lambda \varphi^{i}(x)+\mu(x)=\alpha^{i}(x),
$$

contradicting condition (A-2) for an ex post equilibrium. 
The lemmata above in hand, we can now proceed with the proof of Theorem 1 .

\section{PROOF OF THEOREM 1:}

(Existence) We first prove that both players playing $G^{*}$ is an ex post equilibrium for some constant $\alpha^{*}$. It is easy to verify that given $G^{*}$, the posterior belief is the following function $\varphi^{*}$ for both candidates (so we drop the superscripts indexing candidates):

$$
\varphi^{*}(x)=\left\{\begin{array}{ll}
\frac{\alpha^{*}-\mu(x)}{\lambda} & \text { if } g^{*}(x)>0 \\
1 & \text { if } g^{*}(x)=0
\end{array} .\right.
$$

Accordingly,

$$
\begin{aligned}
\alpha\left(x \mid \varphi^{*}\right) & =\lambda \varphi^{*}(x)+\mu(x) \\
& =\left\{\begin{array}{ll}
\alpha^{*} & \text { if } g^{*}(x)>0 \\
\lambda+\mu(x) & \text { if } g^{*}(x)=0
\end{array} .\right.
\end{aligned}
$$

Noting from (2) that $g^{*}(x)=0$ requires $\alpha^{*}-$ $\mu(x) \geq \lambda$, one sees that conditions (A-1) and (A-2) for an ex post equilibrium are indeed satisfied. It remains only to verify that there is a constant $\alpha^{*}$ which makes $g^{*}$ a density. Since $g^{*}(x) \geq 0$ for all $x \in X$, we need only check that $\int_{X} g^{*}(x) d x=1$ for some $\alpha^{*}$.

Define

$$
\gamma(x, \alpha)=\max \left\{0, \frac{b f(x)}{1-b}\left[\frac{\lambda}{\alpha-\mu(x)}-1\right]\right\}
$$

so that $g^{*}(x)=\gamma\left(x, \alpha^{*}\right)$. We now prove that there is a unique constant $\alpha^{*}$ such that $\int_{X} \gamma(x$, $\left.\alpha^{*}\right) d x=1$. First, observe that $\gamma$ is continuous in $\alpha$. Next, note that if $\alpha \geq \lambda+\mu(m)$, then $\lambda \leq$ $\alpha-\mu(x)$ for all $x \in X$; hence $\gamma(x, \alpha)=0$ for all $x \in X$ and $\int_{X} \gamma(x, \alpha) d x=0<1$. On the other hand, for $\alpha=\mu(m)$ and small $\varepsilon>0$,

$$
\begin{aligned}
& \int_{X} \gamma(x, \mu(m)) d x \geq \int_{m-\varepsilon}^{m+\varepsilon} \gamma(x, \mu(m)) d x \\
& =\int_{m-\varepsilon}^{m+\varepsilon} \max \left\{0, \frac{b f(x)}{1-b}\left[\frac{\lambda}{\mu(m)-\mu(x)}-1\right]\right\} d x
\end{aligned}
$$

$$
\begin{aligned}
& \approx \frac{b f(m)}{1-b}\left[\int_{m-\varepsilon}^{m+\varepsilon} \frac{\lambda}{\mu(m)-\mu(x)} d x-2 \varepsilon\right] \\
& \approx \frac{b f(m)}{1-b}\left[\int_{m-\varepsilon}^{m+\varepsilon} \frac{\lambda}{-\frac{1}{2} \mu^{\prime \prime}(m)(x-m)^{2}} d x-2 \varepsilon\right] \\
& \approx \infty,
\end{aligned}
$$

where the penultimate line follows from $\mu^{\prime}(m)=u_{1}(m, m)=0$ and Taylor expansion, and the last line from the fact that $\mu^{\prime \prime}(m)=$ $u_{11}(m, m) \leq 0$. By the Intermediate Value Theorem, there is a value of $\alpha$, call it $\alpha^{*} \in(\mu(m)$, $\mu(m)+\lambda)$, which satisfies $\int_{X} \gamma\left(x, \alpha^{*}\right) d x=1$. It is straightforward that there cannot be any other value of $\alpha$ such that $\int_{X} \gamma(x, \alpha) d x=1$, since $\gamma(x, \alpha)$ is strictly decreasing in $\alpha$ for any $x$ such that $\gamma(x, \alpha)>0$.

(Uniqueness) Now we prove that both players playing $G^{*}$ is the unique equilibrium. Denote the support of $G^{*}$ by $\left[l^{*}, h^{*}\right] \nsupseteq\{m\}$. Suppose there is another (necessarily ex post, by Lemma A.2) equilibrium where a candidate $i$ plays $G^{i} \neq G^{*}$. By Lemmas A.3 and A.4, $G^{i}$ has a density $g^{i}$ with support $[l, h] \nsupseteq\{m\}$. Since $G^{i} \neq G^{*}$, there must be some nondegenerate interval, $Y \subseteq[l, h]$, such that $g^{i}(x)>g^{*}(x)$ for all $x \in Y$. This implies that $\alpha^{i}(x)<\alpha^{*}(x)$ for all $x \in Y$, and Lemma A.2 then implies $\alpha^{i}(x)<$ $\alpha^{*}(x)$ for all $x \in[l, h]$. Consequently,

$$
\text { (A-3) } \quad g^{i}(x)>g^{*}(x) \quad \text { for all } x \in[l, h] \text {. }
$$

Since $g^{i}$ and $g^{*}$ are both densities, either $l>l^{*}$ or $h<h^{*}$ (or both). Suppose $l>l^{*}$ (the argument is analogous for $\left.h<h^{*}\right)$. By its construction, $g^{*}(x)>0$ for all $x$ in a small neighborhood of $l$. A contradiction ensues with (A-3) if we show that $g^{i}(x) \rightarrow 0$ as $x \rightarrow l$. To prove this, observe that $\varphi^{i}(x)=1$ for all $x<l$, and hence $\alpha^{i}\left(x \mid \varphi^{i}\right)=\lambda+\mu(x)$ for all $x<l$. By condition (A-2), and using Bayes's rule, it must be that for any $\varepsilon>0$, there is a $\delta>0$ such that $g^{i}(x)<\varepsilon$ for all $x \in(l, l+\delta)$.

PROOF OF FACT 5:

Observe that for any $x \in \operatorname{Supp}\left[G^{*}\right]$, when $\lambda \approx \infty$, 


$$
\begin{aligned}
g^{*}(x) & =\frac{b f(x)}{1-b}\left[\frac{\lambda}{\alpha^{*}-\mu(x)}-1\right] \\
& =\frac{b f(x)}{1-b}\left[\frac{1}{\frac{\alpha^{*}}{\lambda}-\frac{\mu(x)}{\lambda}}-1\right] \\
& \approx \frac{b f(x)}{1-b}\left[\frac{\lambda}{\alpha^{*}}-1\right] .
\end{aligned}
$$

The proof is completed by noting from (2) that $\left(\lambda / \alpha^{*}\right) \rightarrow(1 / b)$ as $\lambda \rightarrow \infty$.

\section{PROOF OF FACT 6:}

Let the value $\alpha_{f}^{*}$ refer to the utility generated by $f$ and $\alpha_{h}^{*}$ refer to that generated by $h$. Since $\left[\lambda / \alpha^{*}-\mu(x)\right]-1$ is decreasing as $x$ moves away from the median, $m, h(x)-f(x)$, and $\left[\lambda / \alpha^{*}-\mu(x)\right]-1$ are positively correlated. If the support of $g^{*}$ is the whole policy space,

$$
\begin{aligned}
& \int \frac{b(h(x)-f(x))}{1-b}\left[\frac{\lambda}{\alpha_{f}^{*}-\mu(x)}-1\right] d x \\
& >\int \frac{b(h(x)-f(x))}{1-b} d x \\
& \quad \times \int\left[\frac{\lambda}{\alpha_{f}^{*}-\mu(x)}-1\right] d x=0
\end{aligned}
$$

and thus

$$
\int_{x} \frac{b h(x)}{1-b}\left[\frac{\lambda}{\alpha_{f}^{*}-\mu(x)}-1\right] d x>1 .
$$

It follows that $\alpha_{h}^{*}>\alpha_{f}^{*}$.

\section{PROOF OF FACT 7:}

For the case of $b \rightarrow 1$, inspection of (2) shows that $\alpha^{*}$ increases in $b$ without bound. Since $\operatorname{Supp}\left(g^{*}\right)=\left\{x \mid \lambda+\mu(x) \geq \alpha^{*}\right\}$, as $\alpha^{*}$ increases, the support shrinks to $\{m\}$. (Note that $m$ is always in the support; see Lemma A.4.)

As $b \rightarrow 0$, inspection of (2) shows that $\alpha^{*} \rightarrow$ $\mu(m)$. Thus, indeed $\operatorname{Supp}\left(g^{*}\right) \rightarrow\{x \mid \mu(x)+\lambda \geq$ $\mu(m)\}$. To see that $G^{*}$ converges in distribution to point-mass on $m$, observe that for as $b \rightarrow 0$, for any $\varepsilon>0$, both $\int_{0}^{m-\varepsilon} \max \{0,[b f(x) / 1-$ $\left.\left.b]\left(\lambda / \alpha^{*}-\mu(x)\right)-1\right]\right\} d x \rightarrow 0$ and $\int_{m+\varepsilon}^{1}$ $\max \left\{0,[b f(x) / 1-b]\left[\left(\lambda / \alpha^{*}-\mu(x)\right)-1\right]\right\}$ $d x \rightarrow 0$.

\section{PROOF OF THEOREM 2:}

Throughout, assume that $u$ is convex, i.e., $2 u(0,1)<u(1,1)+u(-1,1)$.

Step 1: First, we show that a Centrist Dominant equilibrium does not exist. As shorthand, let $z \equiv b f_{0}+1-b$. In a Centrist Dominant equilibrium, since both primary voters and the general election voter (strictly) prefer centrists to extremists, strategic candidates must choose the centrist platform, 0 , with probability one, i.e., $g_{0}=1$. Thus, the Bayes update about character given platform 0 is $\varphi(0)=\left(b f_{0} / z\right)$, whereas given platform 1 or -1 it is $\varphi(-1)=$ $\varphi(1)=1$. Note that given this behavior, the probability that a party's nominee is extreme is $(1-z)^{2}$, i.e., it is the probability that both of the party's candidates have character and extreme position. The probability that a party's nominee is centrist is $1-(1-z)^{2}$.

We argue that party $R$ 's voter strictly prefers to nominate an extreme candidate with platform 1 rather than a centrist, which contradicts equilibrium behavior. From the perspective of the $R$ party primary voter, if she nominates a centrist candidate, the general election winner will be centrist, and regardless of which party the general election winner is from, her utility from that candidate is $u(0,1)+\lambda\left(b f_{0} / z\right)$, where we use the fact that the Bayes update about character from centrist candidates of both parties is the same. On the other hand, if she nominates an extreme candidate from her party (if one exists, which is a positive probability event), there are two possibilities: either party $L$ 's nominee is extreme (platform -1 ), which has probability $(1-z)^{2}$; or party $L$ 's nominee is centrist, which has probability $1-(1-z)^{2}$. In the first case, each of the two extreme nominees wins with probability $1 / 2 ; ;^{18}$ in the second case, the centrist from

\footnotetext{
${ }^{18}$ Strictly speaking, the general election voter is indifferent between nominees with platforms -1 and 1 , and need not necessarily randomize uniformly across them. The ensuing argument, however, extends to cover this case because if the randomization favors -1 over 1 to such an extent that the strategic candidate from party $R$ does not have an incentive to deviate to platform 1 , then a strategic
} 
$L$ wins with certainty. Thus, the $R$ primary voter's expected utility from nominating an extremist is

$$
\begin{aligned}
& \frac{1}{2}(1-z)^{2}[(u(1,1)+\lambda)+(u(-1,1)+\lambda)] \\
& \quad+\left(1-(1-z)^{2}\right)\left(u(0,1)+\lambda \frac{b f_{0}}{z}\right) .
\end{aligned}
$$

Therefore, the primary voter has a profitable deviation to nominating an extremist over a centrist if the expression above is strictly greater that the utility from nominating a centrist, $u(0$, 1) $+\lambda\left(b f_{0} / z\right)$. Some algebra reveals that this is equivalent to

$$
u(0,1)<\frac{1}{2}(u(-1,1)+u(1,1))+\lambda \frac{1-b}{z},
$$

which is true since $u$ is convex.

Step 2: Next, we show that the set of parameters for which a General Election Indifference equilibrium exists contains an open set of positive Lebesgue measure. To construct such an equilibrium, we need indifference between centrists and extremists of the general election voter; strict preference for extremists over centrists for party voters; and indifference between platforms for the strategic candidates. Assume that party voters randomize equally over two candidates with the same platform. We consider only party $R$; by symmetry, the logic extends to party $L$. Let $z_{0} \equiv b f_{0}+(1-b) g_{0}$.

For the general election voter to be indifferent between centrists and extremists, we must have

$$
u(0,0)+\lambda \frac{b f_{0}}{z}=u(0,1)+\lambda \frac{b\left(1-f_{0}\right)}{1-z}
$$

or

$(\mathrm{A}-4)$

$$
u(0,0)-u(1,0)=\lambda\left(\frac{b\left(1-f_{0}\right)}{1-z}-\frac{b f_{0}}{z}\right) .
$$

candidate from party $L$ will have an incentive to deviate to platform -1 .
The left-hand side of (A-4) is strictly positive. The right-hand side is continuous and strictly increasing in $z$, where $z$ can range from $b f_{0}$ (if $\left.g_{0}=0\right)$ to $b f_{0}+(1-b)$ (if $g_{0}=$ $1)$. It can be verified that the right-hand side is strictly negative if $z=b f_{0}$ and strictly positive but less than $\lambda\left(1-b / 1-b+b f_{0}\right)$ if $z=b f_{0}+(1-b)$. Thus, so long as $u(0,0)-u(0,1)<\lambda(1-b)$, there is a unique solution $z$ to $(\mathrm{A}-4)$. Note that the solution $z$ pins down $g_{0}$, i.e., the behavior of strategic candidates.

Suppose that if faced with two nominees with platforms -1 and 1 , the general election voter randomizes uniformly over the two; if faced with one centrist and one extremist, she votes for the extremist with probability $p$. Then, the probability that a candidate from party $R$ wins the party nomination and the general election with platform 0 is $1 / 2 z\left(1 / 2 z^{2}+\left(1-z^{2}\right)(1-p)\right)$, where $1 / 2 z$ is the probability that the other candidate within the party is also a centrist, and $z^{2}$ is the probability that the nominee from the other party, $L$, is a centrist. Similarly, the probability of winning with platform 1 is $(z+$ $1 / 2(1-z))\left(p z^{2}+\left(1-z^{2}\right)^{1 / 2}\right)$. These two probabilities of winning must be equal for strategic candidates to randomize between platforms, which solves for $p=1 / 2\left[1-\left(1 / z+z^{2}\right)\right]$. Plainly, $p<1 / 2$, and moreover, for $p \geq 0$ requires that $z+z^{2} \geq 1$, or $z \geq(\sqrt{5}-1 / 2)$. This places a restriction on the set of parameters (recall that $z$ must solve (A-4)), but clearly there is an open and positive measure set of parameters for which $z \geq(\sqrt{5}-1 / 2)$.

It remains only to verify that the $R$ primary voter strictly prefers a candidate with platform 1 to one with platform 0 . Computing the probabilities of various events, this incentive compatibility constraint can be written as

$$
\left(u(0,1)+\lambda \frac{b f_{0}}{z}\right)\left(z^{2}+\left(1-z^{2}\right)(1-p)\right)
$$

$$
+\left(u(-1,1)+\lambda \frac{b\left(1-f_{0}\right)}{1-z}\right)\left(1-z^{2}\right) p
$$

$$
\begin{aligned}
< & \left(u(1,1)+\lambda \frac{b\left(1-f_{0}\right)}{1-z}\right)\left(\frac{1}{2}\left(1-z^{2}\right)+z^{2} p\right) \\
& +\left(u(0,1)+\lambda \frac{b f_{0}}{z}\right) z^{2}(1-p)
\end{aligned}
$$




$$
+\left(u(-1,1)+\lambda \frac{b\left(1-f_{0}\right)}{1-z}\right) \frac{1}{2}\left(1-z^{2}\right) .
$$

Substituting $p=1 / 2\left[1-\left(1 / z+z^{2}\right)\right]$ and the symmetry assumption $u(-1,0)=u(1,0)$ into the inequality above, tedious but straightforward simplification shows that the inequality above is equivalent to

$$
\begin{gathered}
u(0,1)-u(-1,1)-(u(0,0)-u(1,0)) \\
<(u(0,0)-u(0,1)+u(1,1) \\
-u(0,1)) \frac{z}{1-z^{2}} .
\end{gathered}
$$

Since $z \geq 1-z^{2}$ (because $p \geq 0$ ), the inequality above holds if

$$
\begin{aligned}
& u(0,0)-u(1,0)+u(1,1)-u(0,1) \\
& \quad>u(0,1)-u(-1,1)-u(0,0)+u(1,0),
\end{aligned}
$$

which is equivalent to

$$
\begin{gathered}
u(1,1)+u(-1,1)>2 u(0,1) \\
-2(u(0,0)-u(1,0)),
\end{gathered}
$$

which holds because $u$ is convex and $u(0,0>$ $u(1,0)$.

Step 3: To show that a Naive Policy Preference equilibrium exists for an open parameter set of positive measure, one proceeds similarly to the logic of Step 2, but using the appropriate equilibrium conditions: primary voters strictly prefer party extremists over centrists, the general election voter strictly prefers centrists over extremists, and strategic candidates randomize over the center platform and their party extreme. We omit the argument to conserve space; details are available from the authors.

\section{REFERENCES}

Alesina, Alberto. 1988. "Credibility and Policy Convergence in a Two-Party System with Rational Voters." American Economic Review, 78(4): 796-805.

Alesina, Alberto, and Stephen E. Spear. 1988. "An Overlapping Generations Model of Elec- toral Competition." Journal of Public Economics, 37(3): 359-79.

Ansolabehere, Stephen, and James M. Snyder, Jr. 2000. "Valence Politics and Equilibrium in Spatial Election Models." Public Choice, 103(3-4): 327-36.

Ansolabehere, Stephen, and Charles Stewart III. 2001. "Candidate Positioning in U.S. House Elections." American Journal of Political Science, 45(1): 136-59.

Aragones, Enriqueta, and Thomas R. Palfrey. 2002. "Mixed Equilibrium in a Downsian Model with a Favored Candidate." Journal of Economic Theory, 103(1): 131-61.

Banks, Jeffrey S. 1990. "A Model of Electoral Competition with Incomplete Information." Journal of Economic Theory, 50(2): 309-25.

Banks, Jeffrey S., and John Duggan. 2005. "Probabilistic Voting in the Spatial Model of Elections: The Theory of Office-Motivated Candidates." In Social Choice and Strategic Decisions: Essays in Honor of Jeffrey $S$. Banks, ed. David Austen-Smith and John Duggan, 15-56. New York: Springer.

Bernheim, B. Douglas, and Navin Kartik. 2004. "Special Interest Politics and the Quality of Governance." Unpublished.

Besley, Timothy, and Stephen Coate. 1997. "An Economic Model of Representative Democracy." Quarterly Journal of Economics, 112(1): 85-114.

Callander, Steven. 2005. "Political Motivations." Unpublished.

Callander, Steven, and Simon Wilkie. 2005. "Lies, Damned Lies, and Political Campaigns." Unpublished.

Calvert, Randall. 1985. "Robustness of the Multidimensional Voting Model: Candidate Motivations, Uncertainty, and Convergence." American Journal of Political Science, 29(1): 69-95.

Carrillo, Juan, and Micael Castanheira. 2002. "Polarization of Parties and Endogenous Valence." Unpublished.

Downs, Anthony. 1957. An Economic Theory of Democracy. New York: Harper.

Fiorina, Morris P. 1999. "Whatever Happened to the Median Voter?" Unpublished.

Fudenberg, Drew, and Jean Tirole. 1991. Game Theory. Cambridge, MA: MIT Press.

Gerber, Elisabeth R., and Rebecca B. Morton. 1998. "Primary Election Systems and Representation." Journal of Law, Economics, and Organization, 14(2): 304-24. 
Groseclose, Tim. 2001. “A Model of Candidate Location when One Candidate Has a Valence Advantage." American Journal of Political Science, 45(4): 862-86.

Harrington, Joseph E., Jr. 1998. "The Social Selection of Flexible and Rigid Agents." American Economic Review, 88(1): 63-82.

Harsanyi, John C. 1973. "Games with Randomly Disturbed Payoffs: A New Rationale for Mixed-Strategy Equilibrium Points." International Journal of Game Theory, 2(1): 1-23.

Hotelling, Harold. 1929. "Stability in Competition." Economic Journal, 39(153): 41-57.

Kreps, David M., Paul Milgrom, John Roberts, and Robert Wilson. 1982. "Rational Cooperation in the Finitely Repeated Prisoners' Dilemma." Journal of Economic Theory, 27(2): 245-52.

Londregan, John, and Thomas Romer. 1993. "Polarization, Incumbency, and the Personal Vote." In Political Economy: Institutions, Competition, and Representation: Proceedings of the Seventh International Symposium in Economic Theory and Econometrics, ed. William A. Barnett, Melvin J. Hinich, and Norman J. Schofield, 355-77. Cambridge, MA: Cambridge University Press.
Meirowitz, Adam. 2005. "Informational Party Primaries and Strategic Ambiguity." Journal of Theoretical Politics, 17(1): 10736.

Milgrom, Paul R., and Robert J. Weber. 1985. "Distributional Strategies for Games of Incomplete Information." Mathematics of $\mathrm{Op}$ erations Research, 10(4): 619-32.

Osborne, Martin J., and Al Slivinski. 1996. "A Model of Political Competition with CitizenCandidates." Quarterly Journal of Economics, 111(1): 65-96.

Poole, Keith T., and Howard Rosenthal. 1997. Congress: A Political-Economic History of Roll Call Voting. Oxford: Oxford University Press.

Roemer, John E. 1999. "The Democratic Political Economy of Progressive Income Taxation." Econometrica, 67(1): 1-19.

Stokes, Donald E. "Spatial Models of Party Competition." American Political Science Review, 57(2): 368-77.

Wittman, Donald A. 1977. "Candidates with Policy Preferences: A Dynamic Model." Journal of Economic Theory, 14(1): 18089. 